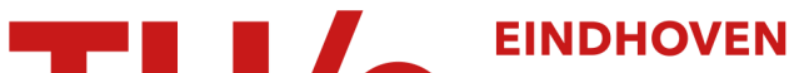 \\ UNIVERSITY OF \\ TECHNOLOGY
}

\section{Recognizing upper body postures using textile strain sensors}

Citation for published version (APA):

Mattmann, C., Amft, O. D., Harms, H., Tröster, G., \& Clemens, F. (2007). Recognizing upper body postures using textile strain sensors. In Proceedings 11th IEEE International Symposium on Wearable Computers, ISWC 2007, 11 October 2007 through 13 October 2007, Boston, MA (pp. 29-36)

https://doi.org/10.1109/ISWC.2007.4373773

DOI:

10.1109/ISWC.2007.4373773

Document status and date:

Published: 01/01/2007

\section{Document Version:}

Publisher's PDF, also known as Version of Record (includes final page, issue and volume numbers)

\section{Please check the document version of this publication:}

- A submitted manuscript is the version of the article upon submission and before peer-review. There can be important differences between the submitted version and the official published version of record. People interested in the research are advised to contact the author for the final version of the publication, or visit the $\mathrm{DOI}$ to the publisher's website.

- The final author version and the galley proof are versions of the publication after peer review.

- The final published version features the final layout of the paper including the volume, issue and page numbers.

Link to publication

\section{General rights}

Copyright and moral rights for the publications made accessible in the public portal are retained by the authors and/or other copyright owners and it is a condition of accessing publications that users recognise and abide by the legal requirements associated with these rights.

- Users may download and print one copy of any publication from the public portal for the purpose of private study or research.

- You may not further distribute the material or use it for any profit-making activity or commercial gain

- You may freely distribute the URL identifying the publication in the public portal.

If the publication is distributed under the terms of Article $25 \mathrm{fa}$ of the Dutch Copyright Act, indicated by the "Taverne" license above, please follow below link for the End User Agreement:

www.tue.nl/taverne

Take down policy

If you believe that this document breaches copyright please contact us at:

openaccess@tue.nl

providing details and we will investigate your claim. 


\title{
Recognizing Upper Body Postures using Textile Strain Sensors
}

\author{
Corinne Mattmann, Oliver Amft, Holger Harms and Gerhard Tröster \\ ETH Zurich, Wearable Computing Lab \\ \{mattmann, amft, harms, troester\}@ife.ee.ethz.ch \\ Frank Clemens \\ EMPA, Materials Science and Technology \\ Frank.Clemens@empa.ch
}

\begin{abstract}
In this paper we present a garment prototype using strain sensors to recognize upper body postures. A novel thermoplastic elastomer strain sensor was used for measuring strain in the clothing. This sensor has a linear resistance response to strain, a small hysteresis and can be fully integrated into textile.

A study was conducted with eight participants wearing the garment and performing a total of 27 upper body postures. A Nä̈ve Bayes classification was applied to identify the different postures. Nearly a complete recognition rate of 97\% was achieved when the classification was adapted to the individual participant. A classification rate of $84 \%$ was achieved for an all-user classification and $65 \%$ for an independent user. These results show the feasibility to recognize postures with our setup, even in an unseen user setting.

Furthermore, we used the garment prototype in a gym experiment to explore its potential for rehabilitation and fitness training. Intensity, speed and number of repetitions could be obtained from the garment sensor data.
\end{abstract}

\section{Introduction}

Clothing is an ideal platform for ubiquitous sensing of user activities with vast applications in sports and personalized health care. Leveraging the full potential of textiles has tremendous advantages with respect to space available, comfortability to the wearer and freedom in number and positioning of sensors. Measuring body postures with textile sensing systems fully utilize those advantages.

Classical posture measurement was limited to permanently equipped rooms because these systems were fixed installations. In recent time, however, many new systems emerged which are portable and can be worn anywhere and anytime. This opens new applications: textile posture and movement sensing can be utilized in fitness training and rehabilitation, e.g. measuring quality and quantity of exercise conduction, as investigated in this work. Another application of portable posture sensing systems is personal prevention, e.g. reporting unfavorable back positions during office work.

In this paper we will present a portable posture measurement setup with textile integrated strain sensors. These sensors measure the strain in a tight-fitting garment caused by body movements. We constructed a prototype of the system and tested it in two experiments. We first performed a study with eight participants conducting 27 postures. In a further experiment, the system was evaluated in a real environment by looking at typical activities in a gym.

After discussing related works in this section, Section 2 is focused on the prototype setup. In particular, the textile strain sensor is analyzed followed by the placement and attachment of the strain sensors to the garment prototype. In Section 3 the posture classification study and results are presented. The gym experiment and analysis is detailed in Section 4. Finally, we conclude with a discussion of the results achieved in this work and give a short outlook.

\subsection{Related work}

Portable systems for posture measurement are based on different kind of sensors. Hansson et al. [7] used $a c$ celerometers to measure joint angles. In [15] and [9] the accelerometers were attached to the pants to detect activities like sitting, standing, walking and lying. Toney [14] used conventional pressure sensors to measure finger joint angles. Dunne et al. [3] measured shoulder and neck movements with a textile piezo-resistive pressure sensor. In [8] a glove was realized using piezoelectric film sensors. Fiberoptics sensors were used in a pressure suit [2] and to measure the seated spinal posture [4]. A commercially available system measuring the posture of the spine is based on ultra- 
sound distance measurements [1]. Measuring upper limb movements with strain gauges was published in [5] by using knit strain sensors and in [13] by using a conductive elastomer. In [6] a textile potential divider was built to measure strain caused by joint movements.

Our approach is similar to the one of Tognetti et al. [13]. We also use a tight-fitting clothing and strain sensors to measure body postures. However, while the above cited paper is focused on upper limb postures, we concentrate on the torso and are able to distinguish between at least 27 different postures (15 sitting and 12 standing postures), covering the whole range of upper body postures. Farringdon et al. concentrate in [5] on the textile integration of their knit strain sensor but do not give any measurement results. The fiberoptic approach of Dunne et al. [4] focuses on measuring the seated spinal posture and is limited to bending back postures (one degree of freedom).

\section{Garment prototype}

A prototype was built (see Fig. 1) with strain sensors attached to the back region of a tight-fitting clothing. These sensors measure strain in the garment caused by different body movements and enable to distinguish between a predefined set of body postures. We used a sensor thread with an elastic range of $100 \%$. More information on the sensor and its fixation to the textile is given in Section 2.1. In Section 2.2 the positioning of the sensors is described. The garment that we used for our prototype was a commercially available catsuit with a front zipper (medium-sized).

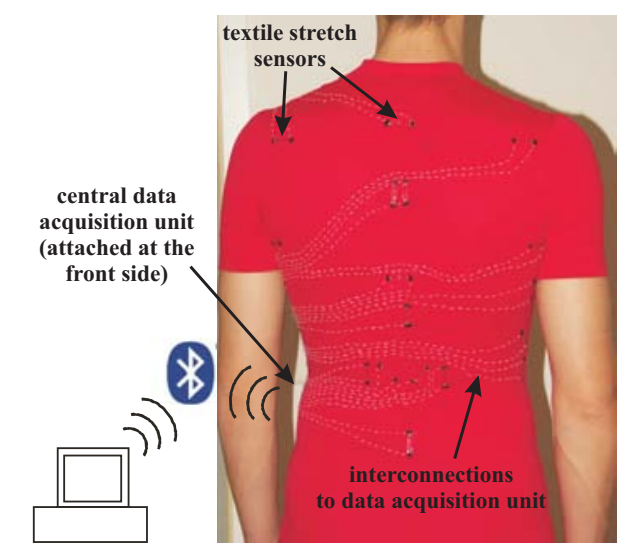

Figure 1. Architecture overview of a prototype recognizing upper body postures using strain sensors.

The sensors were connected to a small data acquisition unit, which digitized (four 8-channel MAX147, 12bit) and transmitted the measured signal to a PC via Bluetooth (MSP430F149, BlueNiceComIII). A graphical user inter- face was implemented which visualized the measured strain values and stored the data into a file. An overview of the setup is given in Fig. 1 [11].

\subsection{Sensor characterization}

A novel strain sensor was used which was developed by EMPA, Switzerland [12]. The sensor thread consists of a commercial thermoplastic elastomer (TPE) filled with $50 \mathrm{wt}-\%$ carbon black powder and changes resistivity with length. It is fiber-shaped with a diameter of $0.3 \mathrm{~mm}$ and has, therefore, the potential to be fully integrated into textile. In this prototype setup, the sensor was attached with a silicone film (see Fig. 2) which enables a measurement range of $100 \%$ strain. The length of the sensor was chosen to be $2 \mathrm{~cm}$.

In this paper, strain is defined as

$$
\text { strain }=\frac{l-l_{0}}{l_{0}} \cdot 100[\%],
$$

whereas $l$ is the current length and $l_{0}$ the initial length.

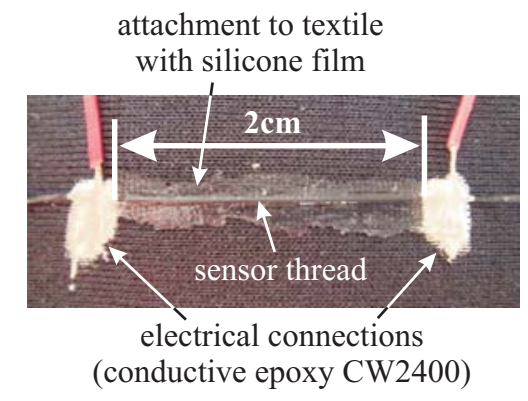
Figure 2. Sensor thread attached to the textile
with a silicone film.

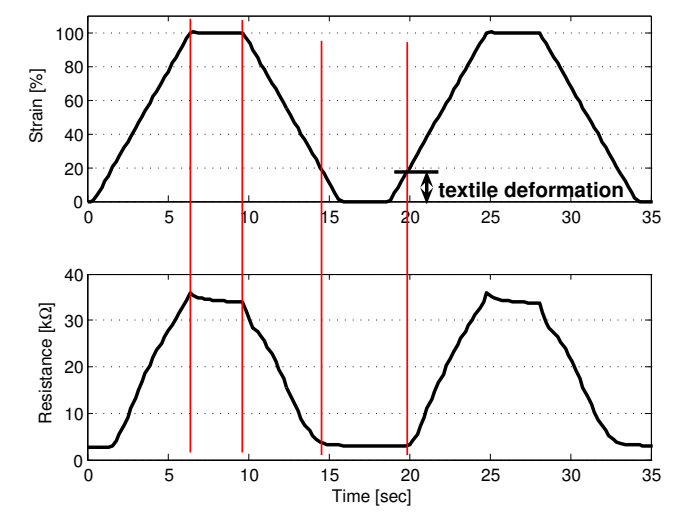

Figure 3. Typical response of sensor to a given strain (sensor length $2 \mathrm{~cm}$ ).

Several measurements were performed in order to characterize the sensor. For these measurements a strain tester 


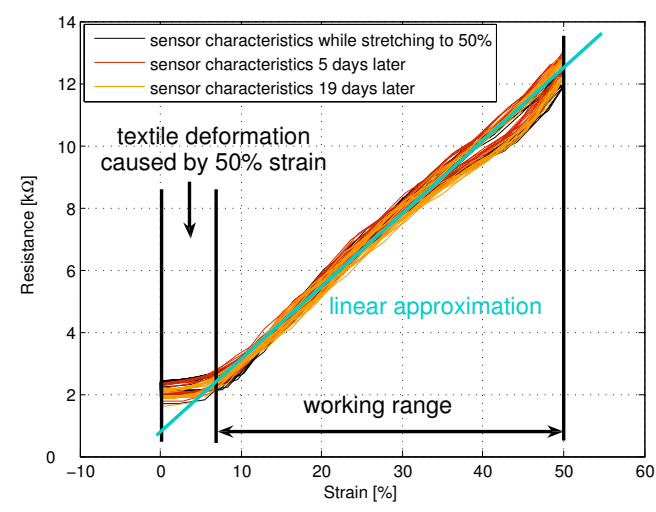

Figure 4. Repeated measurements after 5 and 19 days (sensor length $2 \mathrm{~cm}$, measurement speed $200 \mathrm{~mm} / \mathrm{min}$ ), confirming the long-term stability of the sensor.

was used and the resistance was measured in parallel. Typical measurements were done at a speed of $200 \mathrm{~mm} / \mathrm{min}$ and waiting times at maximal and minimal strain of 3-4sec.

In Fig. 3 a typical resistance vs. time plot is shown. It is apparent that this sensor has a remarkably small overshoot and relaxation time for a textile sensor. A typical resistance vs. strain plot is shown in Fig. 4, indicating a linear rise in resistance when applying strain and only a small hysteresis leading to errors of $\pm 2 \%$ in strain when using a linear approximation. Commercially available strain gauges reach a higher linearity but at a very reduced working range of less than $1 \%$. Our sensor thread has a resistance of approximately $700 \Omega / \mathrm{cm}$ and a high sensitivity ${ }^{1}$ of $2 \mathrm{k} \Omega / \mathrm{mm}$ at a sensor length of $2 \mathrm{~cm}$, enabling a direct measurement of the elongation.

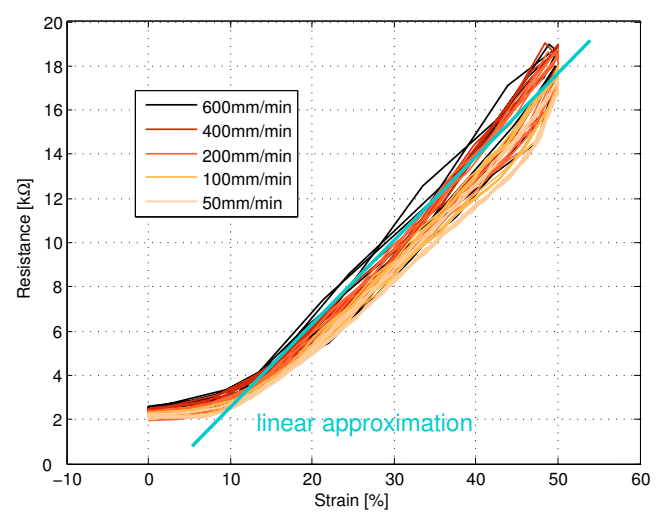

Figure 5. Sensor characteristics at different measurement speeds $(50 \mathrm{~mm} / \mathrm{min}$, $100 \mathrm{~mm} / \mathrm{min}, 200 \mathrm{~mm} / \mathrm{min}, 400 \mathrm{~mm} / \mathrm{min}$ and $600 \mathrm{~mm} / \mathrm{min}$, sensor length $2 \mathrm{~cm}$ ), covering a typical motion speed range of the back.

\footnotetext{
${ }^{1}$ sensitivity $=\frac{\delta R}{\delta l}$
}

The plateau at lower strains in Fig. 4 is caused by a temporary deformation of the textile caused by the large strain applied. This is not a problem for our application as the garment is pre-stretched when worn and we, therefore, use the sensor in the working range only.

To show the stability of the sensor signal, the measurements were repeated after 5 and 19 days. During this time, no drift in the sensor signal was found (see Fig. 4). Increasing the strain velocity $(50 \mathrm{~mm} / \mathrm{min}, 100 \mathrm{~mm} / \mathrm{min}$, $200 \mathrm{~mm} / \mathrm{min}, 400 \mathrm{~mm} / \mathrm{min}$ and $600 \mathrm{~mm} / \mathrm{min}$ ) has shown a marginal rise in resistance. The error increased from $\pm 2 \%$ to $\pm 3.5 \%$ when doing a linear approximation over all five measured speeds (see Fig. 5).

To summarize, the textile strain sensor has the following properties which qualify the sensor for precise strain pattern measurement in a garment:

- small hysteresis,

- linear resistance vs. strain characteristic over a working range of $100 \%$ strain,

- minor dependence on the strain velocity resulting in a total error of $\pm 3.5 \%$,

- stable sensor properties,

- a high sensitivity of $2 \mathrm{k} \Omega / \mathrm{mm}$ (sensor length $2 \mathrm{~cm}$ ),

- thread-like shape.

\subsection{Sensor placement}

The sensor placement was based on reference strain measurements done with an optical motion tracking system [10]. Markers were attached to the back region of a tightfitting clothing and their spatial position was measured with five infrared cameras. By analyzing the distance between the markers, the strain in the clothing could be measured. We performed reference measurements of 12 different postures which served as a basis for the sensor placement. In Fig. 6 an example strain pattern is shown for bending forward.

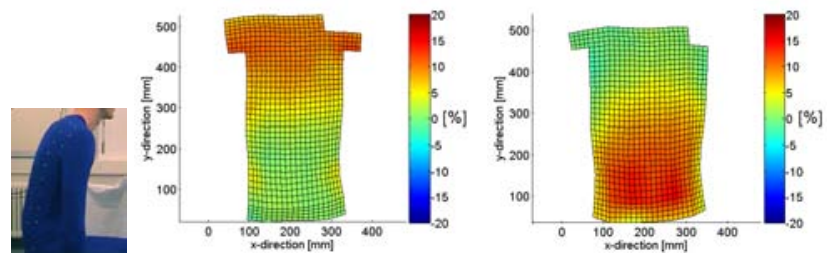

Figure 6. Example strain pattern for bending forward using the reference strain measurement method; middle: strain in horizontal direction; right: strain in vertical direction [10].

When placing the strain sensors on the prototype, our goal was to get a maximal possible coverage of a predefined set of upper torso postures. Hence, we placed sensors on every distinctive position, although, some sensors 
might give redundant information. In Section 3.3 we will show that there is potential to reduce and optimize the number of sensors.

Using the method described above, 21 sensors were placed on the back of a catsuit (see Fig. 7, left).

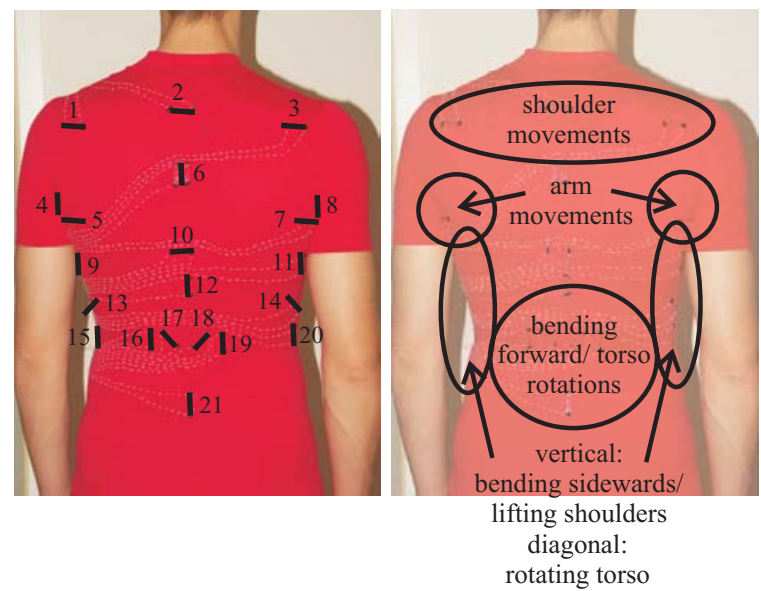

Figure 7. Left: Placement of 21 strain sensors. Right: Regions of elongation for characteristic movements.

As a generalization, the following correlations between movements and elongation in the garment can be given. Additionally, the numbers of the sensors placed to measure each movement are listed (see Fig. 7).

- The region in the upper back is stretched in horizontal direction when the shoulders are moved forward.

$\Rightarrow$ Sensors 1, 2, 3

- Arm movements cause mainly elongations in the armpit region. $\Rightarrow$ Sensors 4, 5, 7, 8, 10

- Bending forward causes elongations in vertical direction, mainly in the lower back region.

$\Rightarrow$ Sensors 6, 12, 16, 19, 21

- Rotations of the torso can be measured in diagonal direction on the back and the side of the torso.

$\Rightarrow$ Sensors 13, 14, 17, 18

- On the side of the torso, we are able to measure bending sidewards and lifting the shoulders when measuring in vertical direction. $\Rightarrow$ Sensors 9, 11, 15, 20

\section{Classification of body postures}

To evaluate the prototype we conducted a study in which the strain sensor readings were used to classify a selection of 27 body postures. A simple classification algorithm was used in combination with a thorough analysis procedure to verify the feasibility of our approach. The following sections present the experimental protocol and summarize the classification method and results.

\subsection{Experimental procedure}

A set of frequently used body postures covering the degrees of freedom in trunk and arm movement were defined. This initial set was adapted to cover both sitting and standing postures resulting in a final set of 15 sitting and 12 standing posture classes. Tab. 1 shows and summarizes the final posture set that was considered in the study. An additional base posture was defined as standing upright with relaxed arms along the body axis. This posture was used for a very basic adaptation of the prototype to the user.

Eight male participants aged between 26 and 31 years were included in the study. The mean body height was $179 \mathrm{~cm}$, standard deviation (SD) $4.1 \mathrm{~cm}$. The mean chest circumference was $95 \mathrm{~cm}(\mathrm{SD} 4 \mathrm{~cm})$ and the waist circumference was $84 \mathrm{~cm}(\mathrm{SD} 4.1 \mathrm{~cm})$.

Participants were instructed to assume the different postures for approx. two seconds in a predefined sequence while wearing the garment prototype. A picture was shown to the participants to indicate each posture, however, postures were not explained or trained beforehand. Each posture was repeated three times and the whole set of postures was recorded twice, so that each posture was recorded six times in total (repetitions 1 to 3 and 4 to 6 are referenced as set 1 and 2 respectively). The complete data set included 1296 postures from the 27 posture classes and eight participants.

The strain sensor values were acquired using a central data acquisition unit attached at the waist level (see Section 2), recording at a sampling rate of $33.5 \mathrm{~Hz}$. An observer annotated the postures during the recordings. The recordings and annotation were reviewed in a post-processing step.

\subsection{Classification methodology}

The strain data of all 21 garment sensors was used as feature set for the posture classifications. In a pre-processing step, the strain was normalized by subtracting the mean sensor value of the base posture from each participant's data set. This method compensates for the variable body compositions and hence a variable strain in the garment among the different participants.

Different classification strategies were analyzed in order to describe the influence of user-dependent training on the discrimination of the different postures. The degree of userdependent training was evaluated by using a user-specific training of the classifier, a training and testing that included observations from all users and a subject-wise leave-oneout split that evaluated the classification performance for a new user of the system.

All classifications were performed on every sample of the data with a Naïve Bayes classifier using a 5-fold cross- 


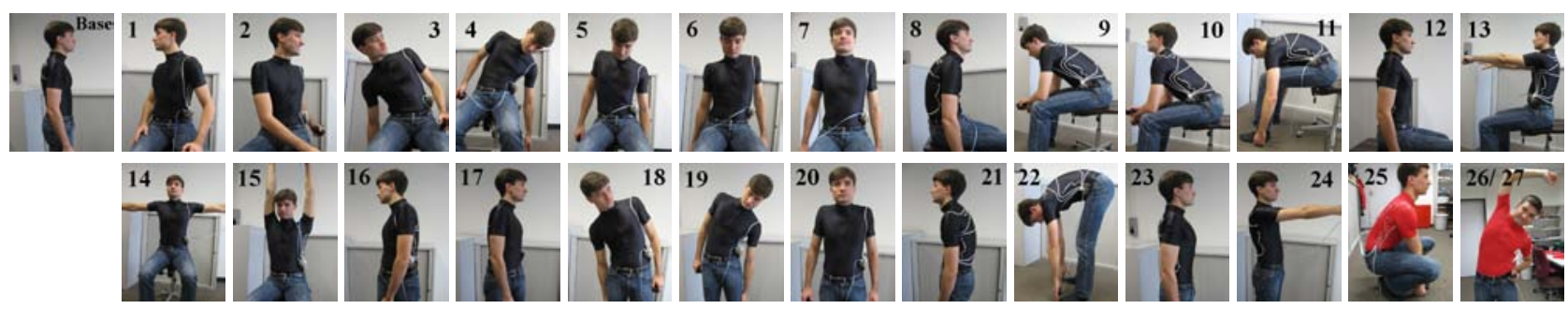

\section{Table 1. Sitting and standing upper body pos- tures included in the study. The postures are visualized at the top of this page.}

\begin{tabular}{l|c|l} 
Base Posture & Class & \\
\hline \hline Standing upright & - & arms relaxed \\
\hline Sitting Postures & Class & \\
\hline \hline Rotation of trunk & 1 & $\begin{array}{l}\text { to the right } \\
\text { to the left }\end{array}$ \\
\hline Bending trunk sidewards & 3 & to the right \\
& 4 & to the left \\
\hline Lifting shoulders & 5 & right shoulder \\
& 6 & left shoulder \\
both shoulders
\end{tabular}

validation procedure to split training and testing observations for the user-specific and all-users evaluations. The splitting procedure was designed to use each observation only once for testing. For the new-user evaluation the data was sliced according to the number of study participants into eight iterations. For each iteration the data from seven of eight participants was used for training and the left out data set for testing.

The class skew due to varying posture lengths was compensated by using an equal number of training samples for all classes. To compare classification results with unequal number of test observations in each class, a normalized accuracy measure was used. The total result of a multi-class classification was derived as mean of the class-relative accuracies (normalized accuracy):

$$
n_{\text {Acc }} \text { Total }=\frac{1}{C} \sum_{i=1}^{C} \frac{\text { Recognized }_{i}}{\text { Relevant }_{i}}
$$

where $C$ is the total number of classes, Recognized R $_{i}$ and Relevant $_{i}$ are the number of correctly identified and the total number of observations in class $i$ respectively.

\subsection{Classification results}

In the user-specific classification an accuracy of 0.97 was achieved with minor confusions of the classes 10 (bending forward) and 25 (squatted) only.

We analyzed the influence of training-testing partitioning by reducing the number of cross-validations from 5 to 2 and consequently, reducing the number of training observations from $80 \%$ to $50 \%$. The accuracy dropped to 0.8 . From this result we concluded that differences exist between the two consecutive posture recording sets (repetitions 1 to $3 \mathrm{com}$ pared to repetitions 4 to 6 ) but only minor variability within each set. Possible reasons for this result are 1) accurate repetition of the postures within each set but slightly different postures between the sets, 2) not enough variance in each set since three repetitions were performed only, 3) sensor inaccuracies and 4) movements of the clothing in comparison to the skin.

Figs. 8 and 9 show the all-user and the new-user classification results respectively (actual class in rows, predicted 


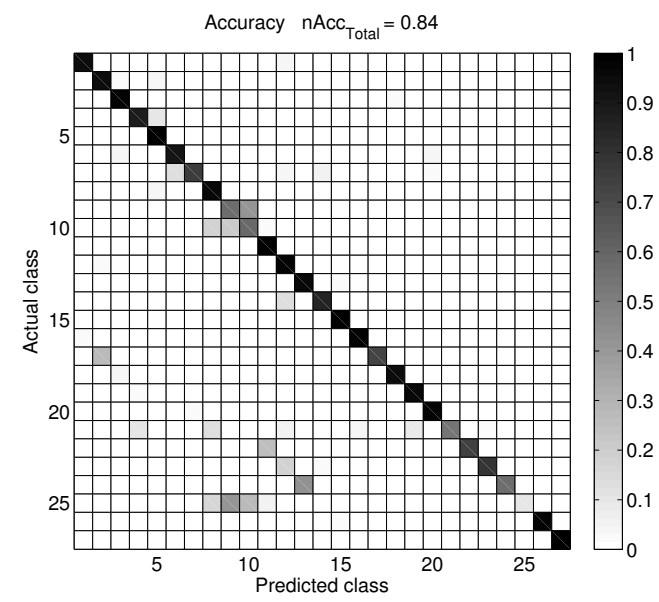

Figure 8. Confusion matrix of the all-user classification of 27 postures.

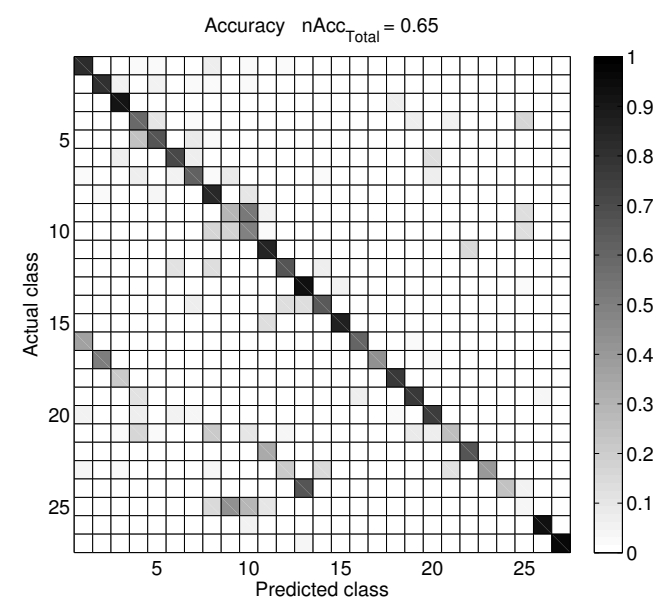

Figure 9. Confusion matrix of the new-user classification of 27 postures.

class in columns). The plot is obtained from the confusion histogram matrix by normalizing each row by the row sum (number of relevant observations for the corresponding class). While the all-user analysis achieved a performance of 0.84 , the performance dropped for the new-user evaluation to 0.65 . A summary of these classification results is shown in Fig. 10, including the minimal and maximal results achieved.

The matrix plots in Figs. 8 and 9 show that certain similar postures could not be discriminated. Two types of error were evident: 1) Confusions appeared between the corresponding sitting and standing postures, e.g. postures 13 and 24 (extending the arms to the front while sitting and standing). 2) Similar postures could not be differentiated like e.g. postures 10, 11 and 25 (bending the trunk forward with a bent and a straight back and squatted). Therefore, the same analysis was repeated for the sitting postures only (postures 1 to 15 ). The results increased from $65 \%$ to $74 \%$

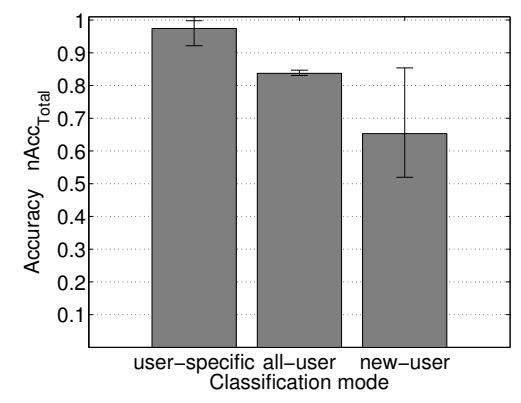

Figure 10. Summary of the classification performances.

for the new-user classification. Hence, we conclude that a posture classification is feasible for many classes even for new-users, however, sitting and standing can hardly be distinguished with the current setup.

To show the potential to optimize the number of sensors, a user-specific classification was performed using 14 empirically chosen sensors (sensor numbers 1, 3, 5, 7, 9, 11, 12, 15-21 in Fig. 7). The result dropped only minimally from $97 \%$ to $96 \%$. Therefore, in a next step we will optimize the number of sensors using for example a Mutual Information approach.

\section{Gym application}

\subsection{Experiment}

In order to show the feasibility of the strain sensitive garment in a real world application, a gym experiment was performed with one person. Another goal was to demonstrate the step from classifying static postures to the recognition of postures during a movement. We performed measurements on the "Rotary Torso", the "Total Abdominal" and the "Lower Back" machines varying speed and intensity (range) of the movements. In the following, we will focus on the Rotary Torso machine (see Fig. 11). The results of the two other machines were similar.

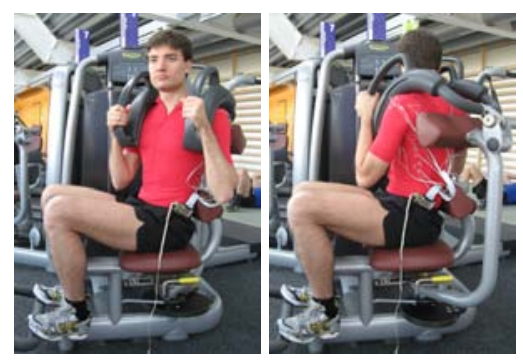

Figure 11. Evaluating the strain sensitive garment in a gym application. Left: Rotary Torso Start Position; Right: Rotary Torso End Position. 
In force training, slow and controlled movements are very important for a balanced training of all muscle groups. If the movement is done too fast (using a momentary impulse), only muscles which are active at the starting position are trained. This makes the training less effective. Another important factor for an intensive training is the usage of the whole movement range so that again as many muscles as possible are trained. With this background information, we measured the following training sequences on the Rotary Torso machine:

- Movement 1: Optimal execution of the exercise with a starting position maximally rotated to the left (see Fig. 11, left) and an end position maximally rotated to the right (see Fig. 11, right). Starting and end position are numbered 1 and 5 respectively for later classification (see Fig. 12).

- Movement 2: Different ranges of movement, starting with a rotation between the starting posture and posture 2 (see Fig. $12,-45^{\circ}$ to $-20^{\circ}$ ) with equally increasing steps to a movement between maximal rotation in both directions (posture 1 to 5 and back to $1,-45^{\circ}$ to $+45^{\circ}$ ). Therefore, 4 different movement ranges were measured.

- Movement 3: Fast performance of the exercise over the whole range of movement where the subject allows his momentum to carry him through the motion.

Additionally, the 5 classes shown in Fig. 12 were recorded separately to be used as training data.

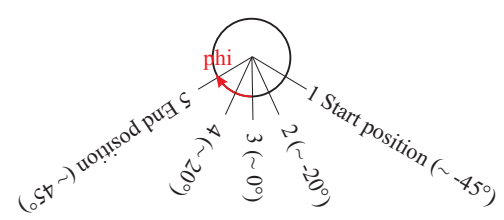

\section{Figure 12. Different ranges of movement on the "Rotary Torso" machine and its assigned class numbers.}

\subsection{Results}

A nearest class center algorithm was used for detection of the exercise posture. The 5 postures shown in Fig. 12 were recorded separately and used as training data. For testing, the continuous data of movements 1 to 3 was used. As movement 1 is equivalent to the last repetition of movement 2 , only results of movements 2 and 3 are shown.

The sensor signals of all 21 sensors were used for classification of which 2 are shown exemplarily in Fig. 13, lower plot. The results of the nearest class center classification are shown in the upper plot. In movement 2 , the rotation angle was gradually increased. This increase was classified correctly in all 4 repetitions. In the subsequent repetitions (movement 3), maximal rotation was performed at high speed. It can be seen that the algorithm classified all the repetitions correctly in the sense that the range of movement was recognized correctly (class 1 through 5). From the classification result, it is even possible to recognize that the first part of the movement was done faster than the second one (going back to the initial position). Based on these results, the speed of the movement, the repetition frequency and the number of repetitions of the training sequence can be calculated.
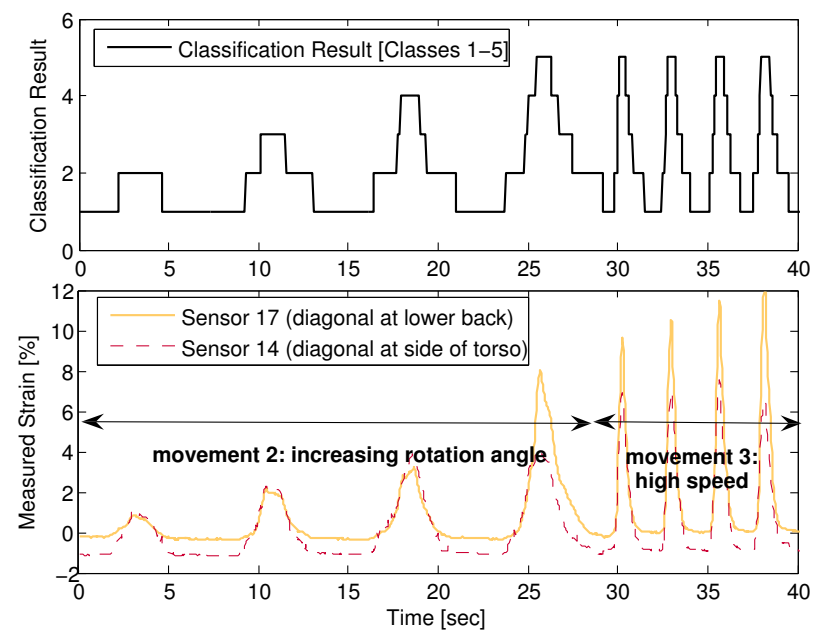

Figure 13. Classification result (upper plot) and 2 example sensors (classification was done with all sensors). First 4 repetitions were performed at normal speed with increasing rotation angle, followed by 4 fast movements using a momentary impulse.

With these results we could show the suitability of the sensorized garment of Fig. 1 to be used in practical applications, exemplarily for a gym exercise. However, this approach could also be used for other applications like e.g. rowing or nordic walking. For the gym task (Rotary Torso) we have shown that the following properties of exercising can be detected:

- Intensity / optimality of training: We are able to distinguish between 4 steps of intensity when the system is trained on the user, so that we can detect whether only part or the whole range of movement is trained.

- Dynamics of movement: The speed of the movement can be measured so that fast and unbalanced movements can be recognized.

- Number of repetitions: The number of repetitions can be recorded and stored as a workout diary. 


\section{Conclusion and outlook}

In this paper a garment prototype to recognize at least 27 upper body postures from 21 strain sensors was presented.

We used a novel textile strain sensor that has a linear resistance vs. strain characteristics and a negligible hysteresis. The resulting measurement error was $\pm 3.5 \%$ over a strain range of up to $100 \%$.

Using this sensor, a garment prototype was developed by attaching the strain sensors with a silicone film in the back region of a tight-fitting garment.

The concept of this setup was proved in a study with eight participants performing 27 postures. Nearly a complete recognition rate of $97 \%$ was achieved with a userspecific training. This demonstrated the feasibility of our design concept. A classification rate of $84 \%$ was obtained for an all-user classification and $65 \%$ for the new user mode. Hence a posture classification is feasible even for an unseen user setting. Two types of error were evident in these classification modes: 1) Confusions appeared between the same sitting and standing postures and 2) similar postures could not be perfectly differentiated. For a reliable discrimination of sitting from standing, additional information would be required like e.g. strain sensors at the waist level or pants.

To our knowledge this is the first time that a garment equipped only with sensor threads has achieved sufficient accuracy to recognize at least 27 upper body postures.

In the future we would like to further pursue the following aspects of our research:

- In this paper we have shown the feasibility of reducing the number of sensors by using a subset of the sensors and achieving similar recognition rates. In a next step we will optimize the number and positioning of the sensors by analyzing their information value, using for example a Mutual Information approach.

- So far, the sensor was attached to the textile with a silicone film. In a next step, we will examine a full integration into the textile.

- Using a simple linear approximation, the sensor thread has an error of $\pm 3.5 \%$. By using a more advanced model, a further reduction of this error is possible.

- A further goal is to proceed from static postures to dynamic movement measurements.

- We have already shown the feasibility of identifying exercise execution on a gym machine. Our measurement concept can easily be transferred to other sports applications like e.g. rowing or nordic walking. We also plan to investigate new application domains like e.g. rehabilitation, safety at work or back protection.

\section{Acknowledgement}

We would like to thank Simon Steiner for the collaboration and for preparing the strain sensors. We are also grateful for the introduction into the gym machines and the professional help of Heiner Iten, sports instructor at the academic sports institution in Zürich (ASVZ).

\section{References}

[1] www.friendly-sensors.de/produkte_gesundheit_sonosens.php.

[2] D. Akin and J. Braden. Neutral buoyancy technologies for extended performance testing of advanced space suits. In 33rd International Conference on Environmental Systems, 2003.

[3] L. Dunne, S. Brady, B. Smyth, and D. Diamond. Initial development and testing of a novel foam-based pressure sensor for wearable sensing. J NeuroEngineering Rehabil, 2(4), 2005.

[4] L. Dunne, P. Walsh, B. Smyth, and B. Caulfield. Design and evaluation of a wearable optical sensor for monitoring seated spinal posture. In Proc. of ISWC, Montreux, 2006.

[5] J. Farringdon, A. J. Moore, N. Tilbury, J. Church, and P. D. Biemond. Wearable sensor badge and sensor jacket for context awareness. In Proc. of ISWC, pages 107-113, 1999.

[6] P. Gibbs and H. Asada. Wearable conductive fiber sensors for measuring joint movements. In IEEE International Conference on Robotics and Automation (ICRA '04), volume 5, pages 4753-4758, 2004.

[7] G. A. Hansson, P. Asterland, N. G. Holmer, and S. Skerfving. Validity and reliability of triaxial accelerometers for inclinometry in posture analysis. Med Biol Eng Comput, 39(4):405-13, 2001.

[8] T. Martin, M. Jones, J. Edmison, T. Sheikh, and Z. Nakad. Modeling and simulating electronic textile applications. ACM Sigplan Notices, 39(7):10-19, 2004.

[9] T. Martin, M. Jones, J. Edmison, and R. Shenoy. Towards a design framework for wearable electronic textiles, 2003.

[10] C. Mattmann, T. Kirstein, and G. Tröster. A method to measure elongations of clothing. In Proc. 1st International Scientific Conference Ambience 05, September 2005.

[11] C. Mattmann and G. Tröster. Design concept of clothing recognizing back postures. In Proc. 3rd IEEE-EMBS International Summer School and Symposium on Medical Devices and Biosensors (ISSS-MDBS 2006), September 2006.

[12] I. Silveira, F. Clemens, C. Bergmann, and T. Graule. Novel strain-sensitive sensors for application in human physiology. In NanoEurope, St. Gallen, 12 - 14 Sep. 2006.

[13] A. Tognetti, F. Lorussi, R. Bartalesi, S. Quaglini, M. Tesconi, G. Zupone, and D. De Rossi. Wearable kinesthetic system for capturing and classifying upper limb gesture in post-stroke rehabilitation. J NeuroEngineering Rehabil, 2(1):8, 2005.

[14] A. Toney. A novel method for joint motion sensing on a wearable computer. In Proc. of ISWC, pages 158-159, 1998.

[15] K. Van Laerhoven and O. Cakmakci. What shall we teach our pants? In Proc. of ISWC, pages 77-83, 2000. 